\title{
Componentes del Ecosistema de Emprendimiento de Lima que Inciden en Crecimiento y Desarrollo de Startups
}

\author{
Karen E. Weinberger Villarán ${ }^{1}$
}

\begin{abstract}
Los ecosistemas de emprendimiento impulsan la competitividad de las startups y estas, a su vez, contribuyen al progreso y bienestar socio-económico. Por ello, el interés en estudiar los componentes del ecosistema de emprendimiento de Lima que inciden en el crecimiento y desarrollo de startups. En febrero 2017, una muestra de 137 startups vinculadas a Innóvate Perú, respondieron un cuestionario. Posteriormente se efectuó un análisis factorial exploratorio - con método ACP- un análisis de clústeres, un ANOVA unidireccional y tablas cruzadas. Se identificaron dos componentes y tres clústeres, caracterizados por la antigüedad y el sector productivo de la startup. Los hallazgos sugieren que la incidencia de los componentes, depende de la fase del proceso de emprendimiento en que se encuentre la startup.
\end{abstract}

Palabras clave: Componentes de ecosistemas de emprendimiento; crecimiento y desarrollo de startups; caracterización de startups; Lima

\section{Components of the Entrepreneurship Ecosystem of Lima that Affect the Growth and Development of Startups}

Abstract: Entrepreneurship ecosystems boost the competitiveness of startups and these, in turn, contribute to socio-economic progress and wellbeing. Therefore, the interest in studying the components of the entrepreneurial ecosystem of Lima that affect the growth and development of startups. In February 2017, a sample of 137 startups linked to Innóvate Peru, answered a questionnaire. Subsequently, an exploratory factor analysis was performed -using the PCA method-, a cluster analysis, a unidirectional ANOVA and cross tables. Two components and three clusters were identified, characterized by the age and the productive sector of the startup. The findings suggest that the incidence of the components depends on the stage of the entrepreneurship process in which the startup is going through.

Keywords: Components of entrepreneurial ecosystems; growth and development of startup ecosystems; startup characterization; Lima

Submitted: March $30^{\text {th }}, 2019$ / Approved: November $4^{\text {th }}, 2019$

\section{Introducción}

Las startups han demostrado ser agentes fundamentales para el desarrollo económico y el bienestar social de una determinada localidad (Mack \& Mayer, 2016), por su capacidad para innovar, romper modelos de negocio tradicionales, generar puestos de trabajo y transformar industrias (Finger \& Samwer, 1998). Sin embargo, para alcanzar el éxito, este tipo de organizaciones requiere de ecosistemas de emprendimiento que faciliten su surgimiento y rápido crecimiento, especialmente en contextos cada vez más globales y competitivos, como el de países emergentes (Hernández \& González, 2016; Roberts et al., 2017).

Estos ecosistemas, con características locales y regionales particulares (Mason \& Brown, 2014), están conformados por diversos elementos -dominios, atributos o componentes- y un conjunto de actores, cuyas acciones y relaciones pueden influir en: i) la intención de emprender (Motoyama y Knowlton, 2016), ii) el proceso de emprendimiento (Shane \& Venkataraman, 2000), iii) el tipo de emprendimiento (Blank, 2003), y iv) el crecimiento y desarrollo de startups (Olutuase, Brijlal, Yan, \& Ologundudu, 2018)the Global Entrepreneurship Monitor (GEM.
El estudio de los ecosistemas de emprendimiento ha despertado el interés de investigadores y académicos (Auerswald et al., 2015; Clarysse, Wright, Bruneel, \& Mahajan, 2014; Spigel \& Harrison, 2018), por sus aportes a la competitividad de las startups y al crecimiento y desarrollo regional (Malecki, 2018). Sin embargo, como señalan Nicotra et al. (2018), la mayoría de los estudios se han centrado en listar los elementos o factores relevantes para el éxito de los ecosistemas de emprendimiento, basándose en la experiencia directa de los investigadores o teorías previas, mas no en estudios o verificaciones empíricas de determinadas localidades o regiones y desde la perspectiva de los emprendedores (Stam \& Spigel, 2016). Además, especialmente en América Latina, la falta de data primaria es uno de los grandes problemas de la región (Lopez \& Alvarez, 2018). Por ello, el interés en desarrollar un estudio empírico que permita identificar los componentes del ecosistema de emprendimiento de Lima que inciden el crecimiento y desarrollo de startups.

El Perú es un país emergente, con una población que supera los 32 millones de habitantes (Instituto Nacional de Estadística e Informática, 2018) y un PBI de aproximadamente US\$ 225 mil millones en 2018 (Banco Central de Reserva del Perú, 2019). En los últimos diez años, los sólidos fundamentos macroeconómicos y el aumento de la

(1) Departamento Académico de Administración, Universidad del Pacífico, Lima, Perú. E-mail: weinberger_ke@up.edu.pe 
Población Económicamente Activa (PEA) de 55\% en 2009 a 56.4\% (aproximadamente 18 millones) (Instituto Nacional de Estadística e Informática, 2019b), le han permitido crecer a un promedio anual de $4.39 \%$ en los últimos diez años y disminuir el índice de pobreza de 33.5\% en 2009 a $20.5 \%$ en 2018 (Instituto Nacional de Estadística e Informática, 2019a).
Por otro lado, como señalan los estudios del Global Entrepreneurship Monitor (GEM), en Perú existe una cultura que promueve, estimula y valora el emprendimiento, lo que se refleja en tasas de emprendimiento en etapas tempranas ${ }^{1}$-TEA por sus siglas en inglés- superiores al promedio global (Bosma \& Kelley, 2019) y entre las tres más altas de la región Latinoamericana (Serida, Alzamora, Guerrero, Borda, \& Morales, 2016), como se observa en las Figuras 1 y 2 . Además, en 2018, ocupó el quinto lugar entre un total de 48 países que participaron en la investigación (Bosma \& Kelley, 2019).

Figura 1. Evolución anual de la actividad emprendedora en etapa temprana (TEA), Perú y mundo: 2009-2018 (\%)

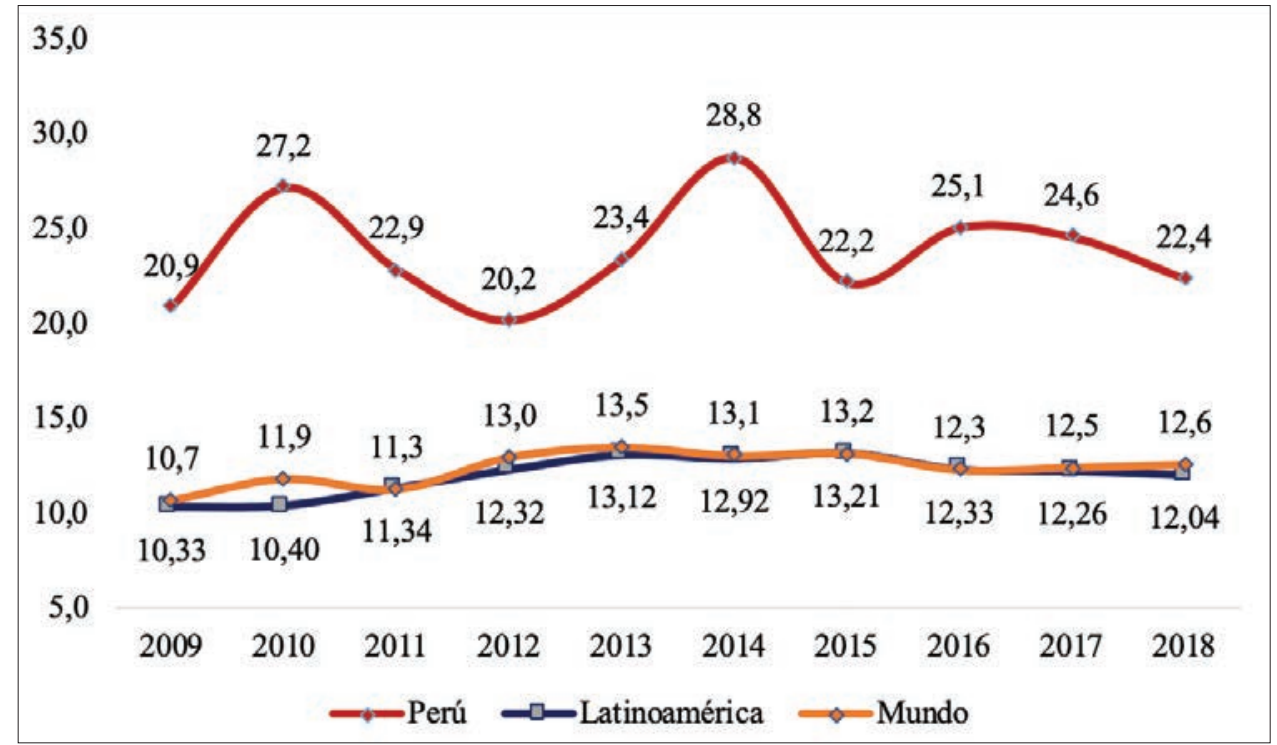

Fuente: Global Entrepreneurship Monitor (Bosma \& Kelley, 2019)

Elaboración propia

Figura 2. Evolución anual de la actividad emprendedora en etapa temprana (TEA), en países de la región con mayores TEA: 2009-2018 (\%)

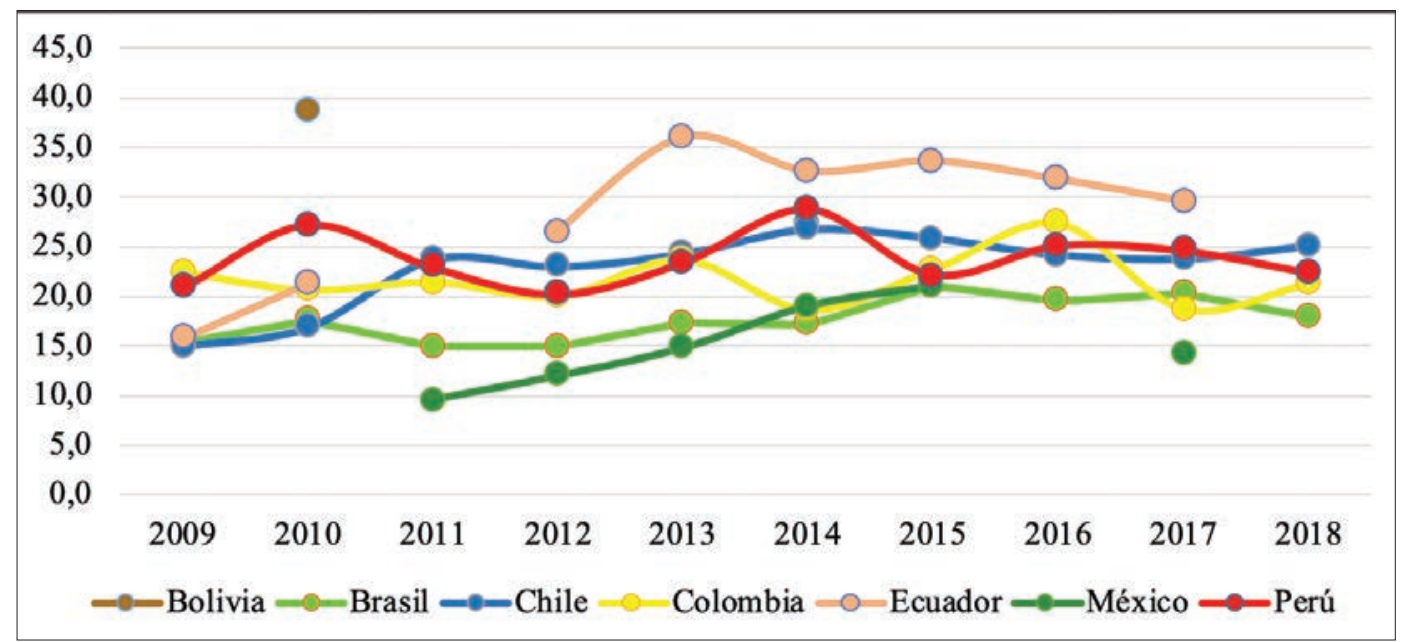

Fuente: Global Entrepreneurship Monitor (Bosma \& Kelley, 2019)

Elaboración propia

\footnotetext{
${ }^{1}$ Índice de Actividad Emprendedora en Etapa Temprana (TEA por sus siglas en inglés). Se refiere al porcentaje de la población, entre 18 y 64 años de edad, que se encuentra activamente en la puesta en marcha de un negocio del cual será dueño o copropietario, o que posee y gestiona un negocio en marcha y que ha pagado sueldos, salarios o cualquier otra retribución a los propietarios por un periodo no mayor a 42 meses (Serida et al., 2018).
} 
Sin embargo, estudios sobre el emprendimiento en América Latina señalan que, a pesar de las condiciones macroeconómicas favorables en el Perú (Kantis, Federico, \& Ibarra García, 2017, 2018), los avances en el desarrollo de políticas públicas en favor del emprendimiento (OCDE, 2016) y las altas tasas de emprendimiento en etapa temprana (Serida et al., 2016; Serida, Guerrero, Alzamora, Borda, \& Morales, 2017), los emprendimientos en el país siguen siendo poco innovadores, tienen bajos niveles de desarrollo tecnológico, muy pocos logran escalar a nivel regional y la mayoría está centralizada en Lima, la capital política. Esta metrópoli concentra el 36.4\% del PBI del país ${ }^{2}$, el 29.2\% de la Población Económicamente Activa (PEA) ${ }^{3}$ (Instituto Nacional de Estadística e Informática, 2019b) y alcanza el 13.1\% del índice de pobreza ${ }^{4}$ (Instituto Nacional de Estadística e Informática, 2019a). Además, concentra el 77\% de las startups del país (OECD, $2016 \mathrm{~b}$ ), el $61.7 \%$ de los proyectos y el $66.7 \%$ de los fondos otorgados por Innóvate Perú, la plataforma de innovación del país (Ministerio de la Producción, 2019). A pesar de ello, la supervivencia, así como el crecimiento y desarrollo de startups en Lima, sigue siendo un reto (Hernández \& González, 2016).

Por lo tanto, dada la importancia de los ecosistemas de emprendimiento para la competitividad de las startups y el bajo nivel de innovación, desarrollo tecnológico y escalabilidad de las startups en Lima, surge el interés por conocer los componentes del ecosistema de emprendimiento de Lima que inciden en el crecimiento y desarrollo de startups, y así contribuir al desarrollo de un marco teórico sobre ecosistemas de emprendimiento.

Para llevar a cabo el estudio, a partir de un cuestionario, se realizó un análisis factorial exploratorio utilizando el método de análisis de componentes principales, un análisis de clústeres, un ANOVA unidireccional y tablas cruzadas para caracterizar a las startups de cada uno de los clústeres identificados.

Los resultados pretenden aportar al escaso número de estudios empíricos sobre componentes de ecosistemas de emprendimiento (Brown, Mawson, \& Mason, 2017; Mason \& Brown, 2014), de países emergentes como Perú (Kantis et al., 2018). Se espera que los resultados sean tomados en consideración por los formuladores de programas e instrumentos en favor del desarrollo de ecosistemas de emprendimiento para startups.

A continuación, se hace una revisión de la literatura sobre startups y ecosistemas de emprendimiento, luego se presenta la metodología empleada para la investigación, los resultados obtenidos y la discusión de los mismos. Finalmente se presentan las conclusiones, recomendaciones e implicancias para formuladores y gestores de políticas públicas.

\section{Revisión de literatura}

El fenómeno del emprendimiento ha pasado de estudiar al emprendedor y su proceso para la creación de nuevas empresas, al estudio de los ecosistemas de emprendimiento que impulsan el surgimiento y desarrollo de un nuevo tipo de organización llamada startup. Sin embargo, si bien en todas las regiones se desarrolla alguna actividad emprendedora y muchas tienen ecosistemas de emprendimiento, no todos los ecosistemas promueven el surgimiento de startups ni contribuyen con servicios para aumentar su competitividad (Roundy, Brockman, \& Bradshaw, 2017), por ello el interés en estudiarlos.

El fenómeno de las startups ha atraído la atención de académicos e investigadores, medios de comunicación, especialistas en innovación, inversionistas y formuladores de políticas públicas, por la relevancia de este tipo de organizaciones en el progreso económico y bienestar de la sociedad (OCDE, 2017). Aunque no se ha llegado a un consenso sobre la definición de startup, Skala (2019) sostiene que todas las definiciones tienen algunas de las siguientes cuatro características: i) se trata de organizaciones jóvenes con recursos financieros escasos; ii) que ofrecen soluciones innovadoras, de manera innovadora y con modelos de negocio innovadores; iii) que, por ser organizaciones ambiciosas, crecen rápidamente; y iv) suelen ser empresas tecnológicas. Además, son consideradas organizaciones de innovación abierta muy poderosas (Spender, Corvello, Grimaldi, \& Rippa, 2017) que cumplen un rol importante en los procesos de innovación.

Por otro lado, el término "sistema de emprendimiento" fue introducido por Spilling (1996), quien sostuvo que el desempeño empresarial estaría afectado por los roles e interacciones entre los actores, así como por aspectos socioculturales, los ciclos económicos, la estructura de las empresas, el análisis de oportunidades, el tipo de actividades empresariales y las características de las relaciones entre los emprendedores. En estos sistemas se darían procesos de intercambio de información, conocimientos y servicios, en condiciones de colaboración y competencia (Spilling, 1996).

En 2006, Cohen introduce el concepto de "ecosistema de emprendimiento", haciendo referencia a un grupo interconectado de actores -universidades, gobierno, profesionales, servicios de apoyo, financiamiento, talento humano- en una comunidad geográfica local, cuyos vínculos formales e informales, infraestructura física y cultura, contribuyen a la sostenibilidad del ecosistema. Es decir, se trata de un conjunto de componentes -diferentes e interdependientes- que interactúan para apoyar la creación de nuevas empresas a través de startups.

Malecki (2018), en su afán por aclarar algunos conceptos, sostiene que los ecosistemas de emprendimiento, a diferencia de los ecosistemas empresariales, clústeres, distritos industriales y sistemas de innovación, centran su estudio en las condiciones para el surgimiento de nuevas iniciativas empresariales y la supervivencia y crecimiento de sus startups. Por lo tanto, el éxito de un ecosistema de emprendimiento debería estar definido por su capacidad para apoyar el entorno regional y el sano crecimiento de las startups, y no solo por las altas tasas de emprendimiento.

\footnotetext{
${ }^{2}$ Equivale a aproximadamente US82 mil millones (Banco Central de Reserva del Perú, 2019).

${ }^{3}$ Aproximadamente 5 millones están en Lima.

${ }^{4}$ De las 9 millones 320 mil personas, que se estima viven en Lima, aproximadamente 1 millón 220 mil se encuentra en situación de pobreza.
} 
Isenberg, uno de los primeros investigadores en proponer un marco de referencia para el estudio de los ecosistemas de emprendimiento (Isenberg, 2010), agrupó decenas de elementos claves de los ecosistemas en trece componentes que consideraba esenciales para los ecosistemas de emprendimiento. Posteriormente, el mismo autor propone seis categorías o dominios. Estos son: i) una cultura que promueva el emprendimiento (I1), ii) la disponibilidad de recursos financieros apropiados para startups (I2), iii) un mercado de clientes suficiente (I3), iv) capital humano de calidad (I4), v) facilidades de infraestructura (I5), y vi) políticas públicas que favorezcan el surgimiento de nuevas iniciativas empresariales (I6) (Isenberg, 2011).
Por otro lado, el Regional Entrepreneurship Acceleration Program del Massachusetts Institute of Technology (MIT REAP por sus siglas en inglés) propone un marco de referencia para el estudio de los ecosistemas de emprendimiento "impulsados por la innovación”, basado en tres pilares fundamentales: i) la capacidad para innovar, es decir la habilidad para desarrollar nuevas tecnologías; ii) la capacidad para emprender, relacionada con la habilidad para lograr el rápido crecimiento de la startup; y iii) el apoyo de instituciones del clúster económico presente en una determinada región (Murray \& Stern, 2015). Este marco de referencia que busca responder a la pregunta: ¿cómo puede una región aprovechar el espíritu empresarial impulsado por la innovación para el progreso económico y social?, identifica seis elementos que contribuyen a promover el emprendimiento y la innovación regional, como se pueden apreciar en la Tabla 1.

Tabla 1. Elementos y actividades del ecosistema de emprendimiento e innovación

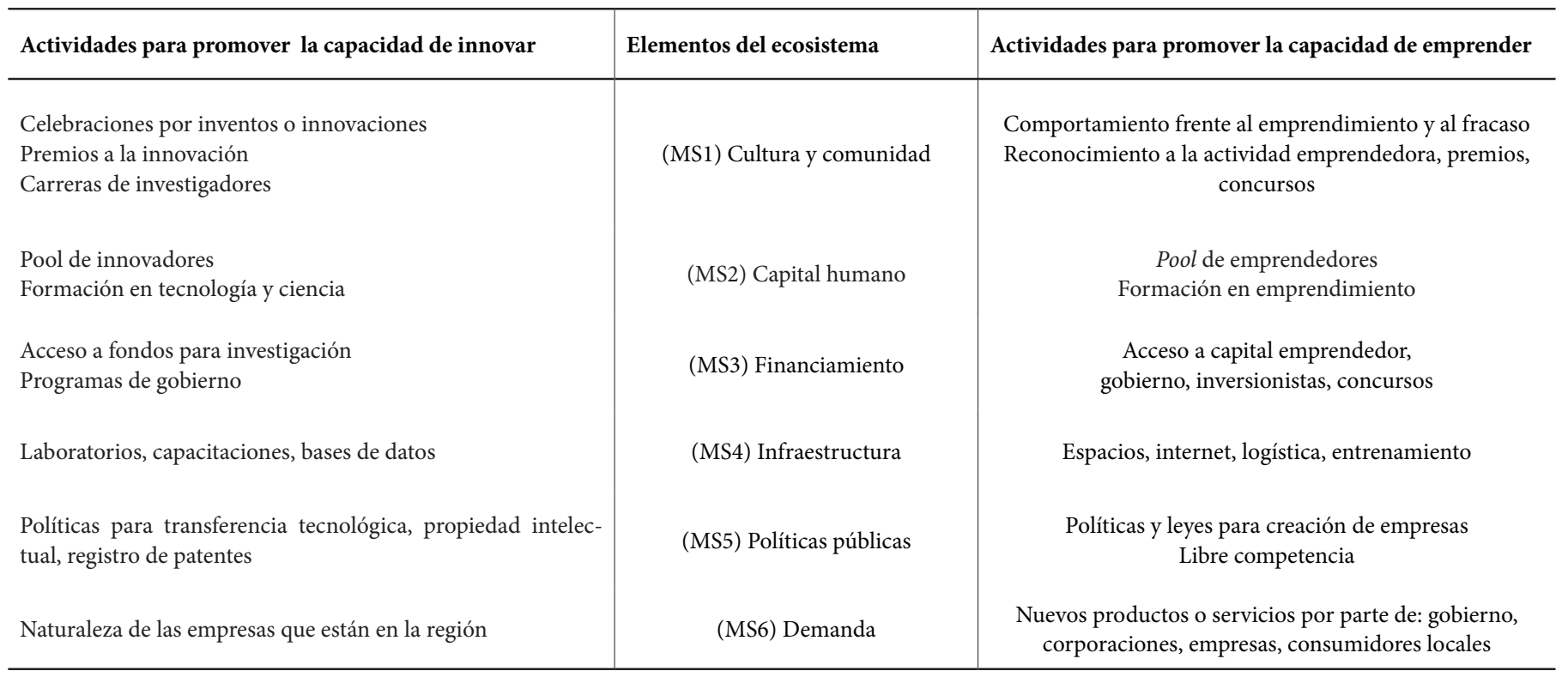

Fuente: Instituto de Tecnología de Massachusetts, Programa Regional de Aceleración de Emprendimiento, cohort 4. (MIT-REAP por sus siglas en inglés)

Elaboración propia

Murray y Stern (2015), integrantes del equipo de profesores e investigadores del MIT REAP, señalan que para el éxito de las startups se requiere, además de emprendedores con capacidad para innovar y emprender, el apoyo y soporte de otros actores, como la academia, las corporaciones, el Estado, los inversionistas y otros emprendedores, cuya experiencia, conocimiento, capacidad de inversión y redes de contacto, aportan al emprendedor e impulsan el emprendimiento basado en la innovación.
Kantis, Federico, Ibarra García y Menéndez (2015) -expertos en emprendimiento latinoamericano- señalan que los ecosistemas para emprendimientos dinámicos están compuestos por diez dimensiones o componentes, asociados a tres factores o ejes conceptuales que promueven o inhiben el desarrollo de emprendimientos dinámicos, como se muestran en la Tabla 2. 
Tabla 2. Ejes conceptuales y dimensiones de los ecosistemas de emprendimiento dinámico

\begin{tabular}{l|l}
\hline Ejes conceptuales & Dimensiones o componentes \\
\hline $\begin{array}{l}\text { Factores que estimulan una masa crítica de } \\
\text { emprendedores }\end{array}$ & (K1) Capital humano emprendedor \\
& $\begin{array}{l}\text { (K2) Sistema educativo } \\
\text { (K4) Cultura local que promueva el emprendimiento innovador } \\
\text { Factores que afectan la existencia de oportu- }\end{array}$ \\
nidades de negocios & (K5) Condiciones de la demanda \\
Factores que promueven o inhiben el desa- & (K7) Plataforma de ciencia y tecnología que permite transformar los conocimientos en innovación \\
rrollo de emprendimientos dinámicos & (K) Ambiente apropiado que facilite las relaciones y redes entre los diversos actores clave como empresarios e \\
& instituciones que faciliten el acceso a recursos (capital social) \\
& (K1) Financiamiento para las diversas etapas del proceso de emprender \\
\hline
\end{tabular}

Fuente: Kantis, Federico, Ibarra García, et al. (2015)

Elaboración propia

Por su parte, Spigel (2015) hace una revisión de la literatura para identificar los elementos que, por su relevancia para el surgimiento, crecimiento y desarrollo de empresas innovadoras, han sido estudiados entre los años 1989 y 2014. Spigel propone clasificar estos elementos en diez atributos, que luego agrupa en tres categorías i) atributos culturales, ii) atributos sociales, y iii) atributos materiales (ver Tabla 3 ).

Tabla 3. Atributos de los ecosistemas de emprendimiento

\begin{tabular}{|c|c|c|}
\hline Tipo de atributo & Atributo & Descripción \\
\hline Cultural & $\begin{array}{l}\text { (Sc1) Cultura de apoyo al emprendimiento } \\
\text { (Sc2) Historia de emprendedores }\end{array}$ & $\begin{array}{l}\text { Normas culturales que promueven y apoyan el emprendimiento } \\
\text { Historias de emprendimientos locales exitosos }\end{array}$ \\
\hline Social & $\begin{array}{l}\text { (Ss1) Redes sociales } \\
\text { (Ss2) Capital emprendedor } \\
\text { (Ss3) Mentores y modelos a seguir } \\
\text { (Ss4) Trabajadores talentosos }\end{array}$ & $\begin{array}{l}\text { Redes sociales que conectan actores y facilitan el uso de los recursos del ecosistema } \\
\text { Disponibilidad de fondos de capital emprendedor (familia, amigos, inversores ángeles, capital } \\
\text { de riesgo) } \\
\text { Exitosos emprendedores locales que son mentores de nuevos emprendedores } \\
\text { Disponibilidad de trabajadores talentosos, deseosos de trabajar en una startup }\end{array}$ \\
\hline Material & $\begin{array}{l}\text { (Sm1) Universidades } \\
(\mathrm{Sm} 2) \text { Servicios de apoyo e infraestructura } \\
\text { (Sm3) Políticas y gobierno } \\
\text { (Sm4) Mercados abiertos }\end{array}$ & $\begin{array}{l}\text { Instituciones académicas que forman emprendedores, crean nuevos conocimientos y desarro- } \\
\text { llan talento para las startups } \\
\text { Incubadoras, aceleradoras, estudios contables y de abogados, oficinas de patentes y transferen- } \\
\text { cia tecnológica. Disponibilidad de oficinas, servicios logísticos, informáticos y de telecomuni- } \\
\text { caciones } \\
\text { Programas del gobierno o regulaciones que apoyan el emprendimiento a través de financia- } \\
\text { miento directo o quitando las barreras a la creación de empresas } \\
\text { Suficientes oportunidades locales para iniciar emprendimientos y que no hayan impedimentos } \\
\text { para acceder mercados globales }\end{array}$ \\
\hline
\end{tabular}

Fuente: Spigel (2015)

Los atributos culturales se basan en las normas o creencias de una determinada región o localidad, con respecto a la actividad de emprender; los atributos sociales se refieren a las relaciones que se dan entre los múltiples actores de los ecosistemas, con el fin de conseguir los recursos necesarios para cada etapa del proceso de emprender; mientras que los atributos materiales se refieren a la existencia de infraestructura o recursos tangibles como son: las universidades, las incubadoras de empresas, los centros de investi- gación, los estudios de abogados, los servicios contables y las políticas públicas en favor de las startups, ya sea para financiar emprendimientos o remover barreras a la creación de nuevas iniciativas empresariales. Spigel y Harrison (2018) sostienen que el tipo de recursos disponibles en un ecosistema (atributos materiales) y la habilidad para que estos recursos fluyan a través de las redes de contacto social (atributos sociales), contribuirán al buen funcionamiento y desarrollo de ecosistemas fuertes. 
Otros autores (Ács, Autio, y Szerb, 2014; Welter, Baker, Audretsch, y Gartner, 2017) afirman que sin una cultura de apoyo al emprendimiento, ni emprendedores con la capacidad para aprovechar las oportunidades generadas en determinadas regiones o localidades, será difícil desarrollar ecosistemas de emprendimiento favorables para el crecimiento y desarrollo de startups. Sin embargo, una cultura emprendedora no asegura el surgimiento, crecimiento y desarrollo de startups, pues este tipo de emprendimientos requieren de ecosistemas que los estimulen a innovar y conseguir los recursos necesarios para ser competitivos, crecer y desarrollarse a nivel global (Murray \& Stern, 2015).

La literatura muestra que, en la última década, el estudio sobre ecosistemas de emprendimiento se ha centrado en identificar los aspectos relevantes para su éxito y los efectos en la actividad emprendedora (Nicotra et al., 2018), pues los elementos de un ecosistema "nutren" a una startup (Tripathi, Seppänen, Boominathan, Oivo, \& Liukkunen, 2019). Sin embargo, dada la variedad aspectos identificados, investigadores y académicos han tratando de categorizarlos en elementos (Aulet, 2008; Fetters, Greene, \& Rice, 2010; Isenberg, 2010; Tripathi, Oivo, Liukkunen, \& Markkula, 2019), dimensiones (Gnyawali \& Fogel, 1994), componentes (Isenberg, 2011; Mack \& Mayer, 2016; Neck, Mayer, Cohen, \& Corbett, 2004; Qian, 2018), factores (Kantis, Federico, \& Ibarra García, 2015; Neck et al., 2004; Spigel, 2015; Stam \& Spigel, 2016; Vogel, 2013), actores (Alvedalen \& Boschma, 2017; Cohen, 2006; Isenberg, 2011; Isenberg \& Onyemah, 2016; Mason \& Brown, 2014; Neck et al., 2004; Roberts et al., 2017; Stam \& Spigel, 2016; Vogel, 2013), niveles
(Theodoraki \& Messeghem, 2017), entre otros, sin llegar a un consenso que permita definir un marco teórico para el estudio de los ecosistemas de emprendimiento. Además, mientras algunos autores se centran en la importancia de crear ecosistemas de emprendimiento para fomentar el surgimiento de nuevas iniciativas empresariales; otros enfatizan en el rol que los actores de estos ecosistemas tienen para impulsar la competitividad de las startups (Auerswald et al., 2015; Clarysse et al., 2014; Spigel \& Harrison, 2018).

Frente a esta falta de consenso y dado que el objetivo del estudio es identificar los componentes del ecosistema de emprendimiento que inciden en el crecimiento y desarrollo de las startups, se optó por la propuesta de Spigel, por ser la que mejor integra estos componentes. Sin embargo, en la Tabla 4, se presentan y comparan las propuestas de Isenberg, Spigel, MIT REAP y Kantis et al., para mostrar que estos también estudian los componentes culturales, sociales y materiales, aunque las dos últimas lo hacen para responder a otra pregunta: ¿cómo puede una región aprovechar el espíritu empresarial impulsado por la innovación para el progreso económico y social? Esta es una pregunta vinculada al estudio de los clústeres, la estrategia y la ventaja competitiva de cada región, más que al estudio de los componentes de un ecosistema de emprendimiento.

Frente a esta falta de consenso, y dadas las similitudes entre los cuatro modelos planteados, se optó por integrarlos en función a los tres tipos de atributos más comunes en la literatura (ver Tabla 4).

Tabla 4. Comparación de los elementos de ecosistemas propuestos por Isenberg (I), Murray \& Stern (MS), Kantis et al. (K) y Spigel (S)

\begin{tabular}{|c|c|}
\hline Atributos sociales & Atributos materiales \\
\hline (I2) Disponibilidad de recursos financieros apropiados para startups & (I3) Mercado de clientes suficiente \\
\hline \multirow[t]{2}{*}{ (I4) Capital humano de calidad } & (I5) Facilidades de infraestructura \\
\hline & (I6) Políticas públicas que favorezcan el surgimiento de nuevas iniciativas empresariales \\
\hline (K4) Condiciones sociales & (K5) Condiciones de la demanda \\
\hline $\begin{array}{l}\text { (K8) Ambiente apropiado que facilite las relaciones y redes entre actores y } \\
\text { acceso a recursos }\end{array}$ & (K6) Estructura empresarial \\
\hline \multirow[t]{2}{*}{ (K9) Financiamiento para las diversas etapas del proceso de emprender } & $\begin{array}{l}\text { (K7) Plataforma de ciencia y tecnología que permiten transformar los conocimien- } \\
\text { tos en innovación }\end{array}$ \\
\hline & (K10) Políticas y regulaciones que fomentan o inhiben actividad emprendedora \\
\hline (Ss1) Redes sociales & (Sm1) Universidades, conocimiento y entrenamiento a emprendedores \\
\hline (Ss2) Disponibilidad de capital para inversión & (Sm2) Servicios de apoyo e infraestructura \\
\hline (Ss3) Mentores y modelos a seguir & (Sm3) Políticas y gobierno \\
\hline (Ss4) Trabajadores talentosos & (Sm4) Mercados abiertos \\
\hline (MS2) El capital humano & (MS4) La infraestructura \\
\hline \multirow[t]{2}{*}{ (MS3) El financiamiento } & (MS5) Las políticas públicas \\
\hline & (MS6) La demanda \\
\hline
\end{tabular}

Atributos culturales

(I1) Cultura que promueva el emprendimiento

(K1) Capital humano emprendedor

(K2) Sistema educativo

(K3) Cultura local que promueva el emprendimiento innovador
(Sc1) Cultura de apoyo al emprendimiento

(Sc2) Historia de emprendedores

(MS1) La cultura y la comunidad 
Es importante señalar que la investigación se enfoca en el estudio de las variables que contribuyen con el crecimiento y desarrollo de startups, mas no en las variables que impulsan el surgimiento de nuevas iniciativas empresariales, pues estudios previos del GEM (Serida et al., 2016) señalan que en Perú existe una una fuerte cultura promotora del emprendimiento, por lo que el atributo cultural no fue analizado.

A continuación, se presenta la metodología empleada para identificar los componentes que, desde la perspectiva de los emprendedores fundadores de startups, han tenido mayor influencia en el crecimiento y desarrollo de sus startups.

\section{Metodología}

Dado que el estudio de los ecosistemas de emprendimiento es un fenómeno complejo (Roundy, Bradshaw, \& Brockman, 2018) que hasta el momento no ha sido suficientemente expuesto a la comunidad de investigadores, especialmente en países latinoamericanos (Lopez \& Alvarez, 2018), se decidió explorar y comprender el fenómeno a través de un estudio de caso (Yin, 2013). Se eligió el caso de las startups de Innóvate Perú. Innóvate Perú es el Programa Nacional de Innovación para la Competitividad y Productividad, que busca incrementar la innovación productiva, impulsar el emprendimiento innovador y fomentar la absorción y adaptación de tecnologías para las empresas (Ministerio de la Producción, 2014). Se eligió este caso porque Innóvate Perú es la plataforma del Estado que, desde 2014, fomenta y financia la innovación en el Perú, tiene mayor cercanía con las startups -indistintamente del tamaño o sector al que pertenezcan-, responde a las necesidades de ellas en sus distintas fases de desarrollo y fortalece a los actores del ecosistema de emprendimiento para startups.

En el marco del MIT REAP, un programa de dos años que busca mejorar los ecosistemas regionales de emprendimiento basados en innovación, el equipo de investigación del MIT REAP Lima-cohort $4^{5}$ desarrolló un cuestionario en base a la revisión de la literatura y a estudios exploratorios previos del ecosistema de emprendimiento de Lima (Hernández \& González, 2016; Kantis, Federico, Ibarra García, et al., 2015), con el fin de conocer cómo se venía desarrollando el ecosistema de emprendimiento peruano y qué oportunidades de mejora existían. Dicho instrumento de medición fue aplicado en una encuesta piloto a 25 emprendedores de alto impacto ${ }^{6}$ registrados en la base de datos de Innóvate Perú, y validado cualitativamente por 12 expertos en ecosistemas de emprendimiento e innovación 7 . Tratándose de un estudio exploratorio, cuya escala de medida no había sido validada anteriormente, se realizó un análisis de convergencia para corroborar la validez del instrumento.
El cuestionario fue distribuido virtualmente en febrero de 2017 a la base de datos de emprendedores que alguna vez habían postulado a los fondos para la innovación de Innóvate Perú, pues es la que mayor número de startups congrega dada su misión institucional. Se obtuvieron 296 respuestas, de las cuales 137 fueron consideradas válidas para los propósitos de nuestro estudio por las siguientes razones: i) quien respondió la encuesta había fundado y se encontraba gestionando el emprendimiento, ii) el encuestado consideraba que su emprendimiento era innovador y tenía un componente tecnológico, iii) conocía los fondos para la innovación y el emprendimiento de Innóvate Perú, y iv) respondió a la pregunta: “¿qué acciones han influido en el crecimiento y desarrollo de su startup?"

Para medir la influencia de cada una de las 12 variables, se empleó la escala de Likert 1-7, siendo 1 "Nada influyente" y 7 "Totalmente influyente". Se calculó la media (M) como tendencia central y la desviación estándar (DS) como medida de dispersión o de variabilidad, para cada una de las variables planteadas en el cuestionario.

Para comprobar si el tamaño de la muestra era suficiente, el método elegido era el correcto y si el instrumento para el recojo de información soportaba las pruebas de confiabilidad y validez, se utilizaron el índice Kayser-Meyer-Olkin (KMO), la prueba de esfericidad de Barlett, y se estimaron el Alfa de Cronbach, la Varianza Extraída Promedio ( $A V E$ por sus siglas en inglés).

Posteriormente, se realizó un Análisis de Componentes Principales (ACP) para determinar a qué componentes corresponden cada una de las variables utilizadas en el estudio, luego un análisis de clústeres jerárquicos con el método de Ward en base a los componentes identificados, y un análisis ANOVA unidireccional para realizar una diferenciación de las medias entre las agrupaciones obtenidas por el análisis de clústeres. Finalmente se realizaron dos tablas cruzadas, que relacionan a la variable de clústeres con cada una de las siguientes variables: "antigüedad de la empresa" y "sector productivo".

A continuación, se presentan los resultados del estudio.

\section{Resultados}

\section{Estadísticos descriptivos}

Como se puede observar en la Tabla 5, las dos variables más valoradas por los emprendedores son: la existencia de un mercado atractivo $(M=5.16)$ y el acceso a tecnología $(M=4.73)$, siendo las únicas dos variables con una media superior a 4 .

\footnotetext{
${ }^{5}$ En esta cuarta promoción (2016 - 2018) participaron seis ciudades: Dubai, Islandia, Laos, Lima, Madrid y Nova Scotia. Cada ciudad estuvo representada por una delegación de funcionarios de: el gobierno, la academia, los emprendedores, los inversionistas y las grandes empresas. Los representantes del Perú fueron: Gonzalo Villarán Córdova (inicialmente representante de UTEC Ventures y posteriormente del Ministerio de la Producción) y Gonzalo Villarán Elías (Innóvate Perú), ambos en representación del Estado; Luis Terrones Morote y Desiree Alayza (COFIDE) y Gonzalo Begazo (Inversionista) en representación del financiamiento; Giancarlo Seco (ISEND) en representación de los emprendedores; Alessandra Corrochano (INTERCORP), Martín Ferraro (Grupo Hochschild) y Miguel Paredes (Grupo Breca) en representación de las corporaciones; José Deustua (UTEC Ventures) y Karen Weinberger (Universidad del Pacífico), ambos en representación de la academia.

${ }^{6}$ Innóvate Perú considera como emprendedor de alto impacto a aquel que emprendimiento innovador, que crece rápidamente y genera altos ingresos pues cubre una necesidad o aprovecha una oportunidad de mercado importante (Ministerio de la Producción, 2015).

${ }^{7}$ Diez en el Perú y dos en el extranjero.
} 
Tabla 5. Estadísticos descriptivos de las variables valoradas por emprendedores

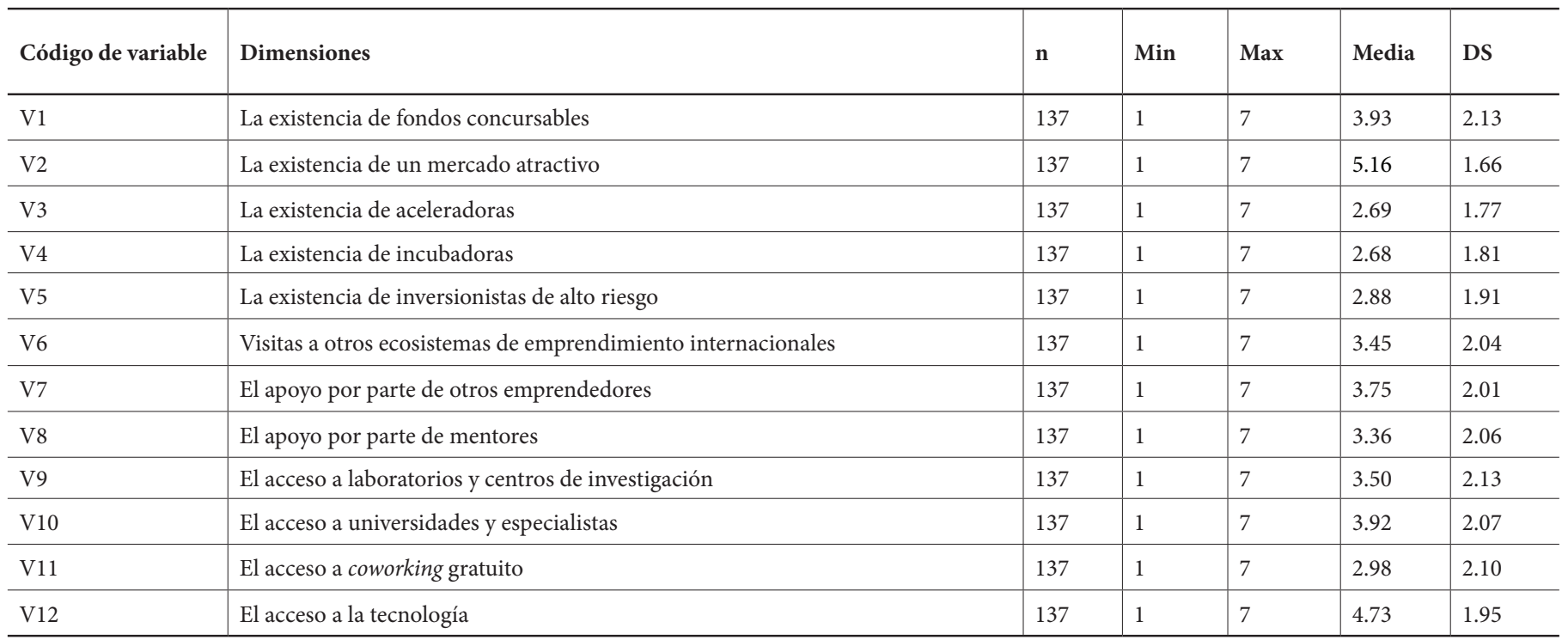

Fuente: encuesta aplicada entre el 14 y 21 de febrero de 2017.

Por el contrario, las cuatro variables con valoraciones promedio inferiores a 3 son: la existencia de incubadoras $(M=2.68)$, la existencia de aceleradoras $(\mathrm{M}=2.69)$, la existencia de inversionistas de alto riesgo $(\mathrm{M}=2.88)$ y el acceso a coworking gratuito $(\mathrm{M}=2.98)$.

Análisis de Componentes Principales

Al emplear el método de Análisis de Componentes Principales (ACP), se encontró que el índice Kayser-Meyer-Olkin (KMO) equivale a $89.6 \%$, lo que indica que el tamaño muestral es adecuado para el análisis. Además, la prueba de esfericidad de Barlett (aprox. Chicuadrado $=1029.35$; g.l. $=66$; valor- $\mathrm{p}=.000$ ) señala que la matriz de correlaciones no es una matriz identidad, por lo que el método elegido es el correcto.

Como se muestra en la Tabla 6, se identificaron dos componentes, cuyos coeficientes Alfa de Cronbach fueron 0.91 y 0.83 superando el valor aceptado de 0.70 (Nunnally, 1994), lo que muestra el alto grado de confiabilidad que presenta cada componente. En conjunto, ambos componentes brindan un porcentaje de varianza acumulado de $65.09 \%$ que supera el valor de $60 \%$ de la varianza total señalado como satisfactoria en ciencias sociales por Hair Jr, Anderson, Tatham, \& Black, (1999, p. 93).

Tabla 6. Matriz de Análisis Componentes Principales

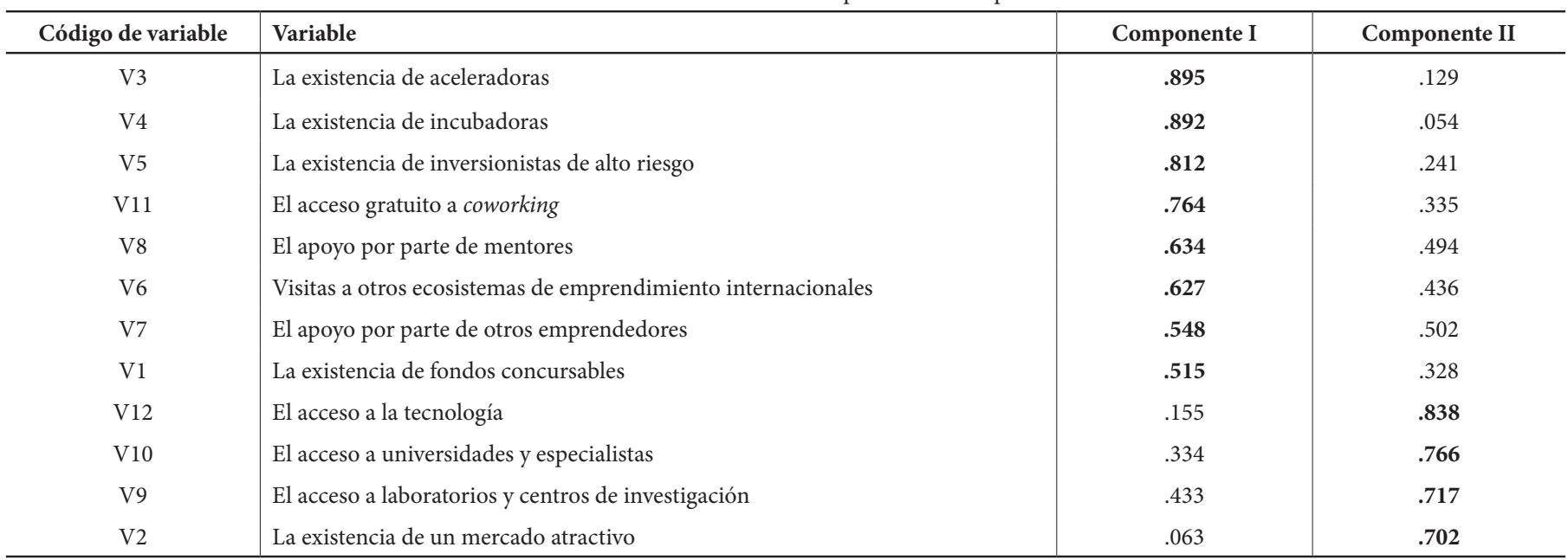

Tal como se observa en la Tabla 6, las variables que conforman el Componente I se asocian con los atributos sociales, en tanto que las variables que conforman el Componente II pueden denominarse como atributos materiales. Por ello, en adelante, dichos componentes se denotan como "Componente social" y "Componente material", respectivamente. 
Análisis de clústeres por el método de Ward

Posteriormente, se realizó un análisis de clústeres jerárquico con el método de Ward en base a los dos componentes hallados. Los resultados muestran tres clústeres: el clúster 1 (C1) está compuesto por 55 startups, y los clústeres 2 (C2) y 3 (C3), por 41 startups cada uno.
Se tomó la decisión de categorizar a los clústeres en función a la etapa del proceso de emprendimiento pues, cada fase en el desarrollo de una startup, tiene distintas necesidades (OCDE, 2016; Spigel \& Harrison, 2018) y, por lo tanto, se asume que requerirá distintos componentes para su crecimiento y desarrollo. Así, el primer clúster se denomina "startups en etapas iniciales" (CI); el segundo, "startups en etapa de supervivencia" (CS); y el tercero, "startups en etapa de crecimiento" (CC).

Tabla 7. Caracterización de los clústeres en función a medias de los componentes

\begin{tabular}{|c|c|c|c|}
\hline Denominación de clúster & Número de casos & $\begin{array}{c}\text { Componente I } \\
\text { (Medias) }\end{array}$ & $\begin{array}{c}\text { Componente II } \\
\text { (Medias) }\end{array}$ \\
\hline C1: Startups en etapas iniciales (CI) & 55 & $4.79 / 7$ & $5.29 / 7$ \\
\hline C2: Startups en etapa de supervivencia (CS) & 41 & $2.11 / 7$ & $2.49 / 7$ \\
\hline C3: Startups en etapa de crecimiento (CC) & 41 & $2.21 / 7$ & $4.87 / 7$ \\
\hline
\end{tabular}

Se cruzaron los dos componentes obtenidos con los tres clústeres (ver Tabla 7). Considerando que el máximo puntaje que podía recibir cada variable del cuestionario fue 7 puntos, se observa que el CI puntúa positivamente en ambos componentes, el CS puntúa negativamente en ambos componentes, mientras que el CC puntúa positivamente en el componente material y negativamente en el social, lo que se aprecia con mayor claridad en la Figura 3.

Figura 3. Medias de los clústeres según componentes

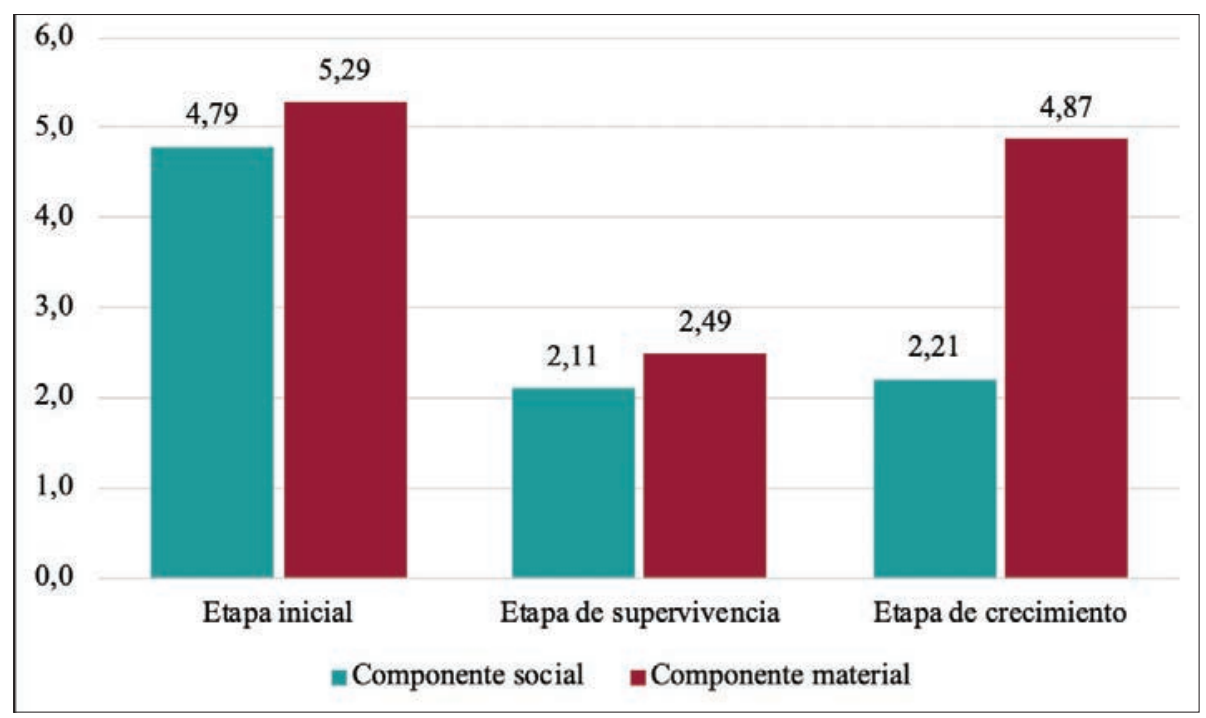

Para realizar una diferenciación de medias entre las tres agrupaciones obtenidas por el análisis de clústeres, se realizó un análisis ANOVA unidireccional, el que cumplió con los supuestos de homogeneidad de varianzas. Se encontró que para el Componente social existe una diferenciación de medias entre las tres agrupaciones $(\mathrm{F}=161.184 ; \mathrm{p}$ $<0.01)$, así como también para el Componente material $(\mathrm{F}=95.586$; $\mathrm{p}<0.01)$.

Al realizar el análisis post-hoc de Tukey, como se observa en Tabla 8, los clústeres de "startups en etapa de supervivencia" $(\mathrm{M}=2.11)$ y "startups en etapa de crecimiento" ( $\mathrm{M}=2.21)$ no poseen una diferenciación significativa de medias para el componente I, siendo el clúster de "startups en etapa inicial" el que mayor puntúa en este componente $(\mathrm{M}=4.79)$. Sin embargo, en el caso del componente II, los clústeres de "startups en etapa inicial" ( $\mathrm{M}=5.29)$ y "startups en etapa de crecimiento" $(\mathrm{M}=4.87)$ no poseen diferenciación significativa de medias y puntúan más que el clúster de "startups en etapa de supervivencia" $(\mathrm{M}=2,49)$.

\section{Tablas cruzadas}

Se desarrollaron tablas cruzadas con el fin de analizar los perfiles derivados de los componentes identificados y caracterizar a las startups en función de la "antigüedad de la empresa" (chi-cuadrado = 19.125; g.l. $=8 ; \mathrm{p}<0.05$ ) y el "sector productivo" (chi-cuadrado = 20.533; g.l. $=12 ; \mathrm{p}<0.10)$ al que pertenecen. 


\section{a) Antigüedad de la empresa}

En la Tabla 8 se observa que, en el CI, la mayoría de las startups (30 de un total de 55) tienen tres o menos años de antigüedad, es por ello que se decidió denominarlo "startups en etapa inicial" (SEI). En el CC, la mayoría de las startups (24 de un total de 41) tiene más de seis años de antigüedad, por lo cual se le denominó "startups en etapa de crecimiento" (SEC). Finalmente, al CS se le denominó el "startups en etapa de supervivencia" (SES) por ser la etapa intermedia del proceso de emprender de toda startup.
Como se observa en la Tabla 8, el 74.5\% (41 de 55) de las startups del CI tienen cinco o menos años de antigüedad. Para el caso de las startups en etapa de supervivencia, no se visualiza un patrón con respecto a la antigüedad de las mismas. De las 41 startups en este clúster, $14.6 \%$ (6) tienen menos de un año; $29.3 \%$ (12) tienen entre uno y tres años, $12.2 \%$ (5) tienen entre tres y cinco años, $29.3 \%$ (12) entre seis y diez años, y 14.6\% (6) tiene más de diez años. Del clúster de "startups en etapa de crecimiento", el 58.5\% (24 de un total de 41) tienen seis o más años de antigüedad.

Tabla 8. Tabla cruzada de la Antigüedad de la empresa y Clústeres

\begin{tabular}{|c|c|c|c|c|c|}
\hline \multicolumn{6}{|c|}{ Tabla cruzada "antigüedad de empresa" ${ }^{*}$ Clústeres } \\
\hline \multirow[b]{2}{*}{ Número de años } & & \multicolumn{3}{|c|}{ Startups agrupadas por clústeres } & \multirow{2}{*}{$\begin{array}{l}\text { Total de empresas } \\
\text { por "antigüedad" }\end{array}$} \\
\hline & & “Startups en & "Startups en etapa de & "Startups en etapa & \\
\hline \multirow{2}{*}{$\begin{array}{l}\text { Menos de uno } \\
(21)\end{array}$} & Recuento & 12 & 6 & 3 & 21 \\
\hline & $\%$ del total & $8.8 \%$ & $4.4 \%$ & $2.2 \%$ & $15.3 \%$ \\
\hline \multirow{3}{*}{$\begin{array}{l}\text { Entre uno y tres } \\
(35)\end{array}$} & Recuento & 18 & 12 & 5 & 35 \\
\hline & $\%$ dentro de Antigüedad de empresa & $51.4 \%$ & $34.3 \%$ & $14.3 \%$ & $100.0 \%$ \\
\hline & $\%$ dentro de Clústeres & $32.7 \%$ & $29.3 \%$ & $12.2 \%$ & $25.5 \%$ \\
\hline \multirow{2}{*}{$\begin{array}{l}\text { Entre tres y } \\
\text { cinco }(25)\end{array}$} & $\%$ dentro de Clústeres & $20.0 \%$ & $12.2 \%$ & $22.0 \%$ & $18.2 \%$ \\
\hline & $\%$ del total & $8.0 \%$ & $3.6 \%$ & $6.6 \%$ & $18.2 \%$ \\
\hline \multirow{4}{*}{$\begin{array}{l}\text { Entre seis y } \\
\text { diez (34) }\end{array}$} & Recuento & 5 & 12 & 17 & 34 \\
\hline & $\%$ dentro de Antigüedad de empresa & $14.7 \%$ & $35.3 \%$ & $50.0 \%$ & $100.0 \%$ \\
\hline & $\%$ dentro de Clústeres & $9.1 \%$ & $29.3 \%$ & $41.5 \%$ & $24.8 \%$ \\
\hline & $\%$ del total & $3.6 \%$ & $8.8 \%$ & $12.4 \%$ & $24.8 \%$ \\
\hline \multirow{3}{*}{$\begin{array}{l}\text { Más de diez } \\
\text { (22) }\end{array}$} & Recuento & 9 & 6 & 7 & 22 \\
\hline & $\%$ dentro de Antigüedad de empresa & $40.9 \%$ & $27.3 \%$ & $31.8 \%$ & $100.0 \%$ \\
\hline & $\%$ dentro de Clústeres & $16.4 \%$ & $14.6 \%$ & $17.1 \%$ & $16.1 \%$ \\
\hline
\end{tabular}

Si se analizan las startups en función a su antigüedad, de las 56 empresas que tienen tres o menos años de antigüedad, el 14.3\% (8) pertenecen al CC, el 32.1\% (18) pertenecen al CS y el 53.6\% (30) pertenecen al CI. De las 25 empresas que tienen entre tres y cinco años de antigüedad, el $44 \%$ (11) pertenecen al CI, el 36\% (9) al CC y el 20\% (5) al CS. Por otro lado, de las 34 startups que tienen entre seis y diez años de antigüedad, el 50\% (17) pertenecen al CC, el 35.3\% (12) al CS y $14.7 \%$ (5) al CI. Finalmente, de las 22 startups que tienen más de diez años de antigüedad, el 31.8\% (7) pertenecen al CC, $27.3 \%$ (6) al CS, mientras que el $40.9 \%$ pertenece CI. Cabe resaltar que, para todas aquellas startups que tienen tres o más años de antigüedad, el número de "startups en etapa de crecimiento" es mayor que el número de "startups en etapa de supervivencia". 
Por otro lado, la mayoría de las startups con cinco o menos años de antigüedad, se encuentran en el clúster de "startups en etapa inicial" y lo mismo sucede con las startups con más de diez años de antigüedad. Finalmente, la mayoría de las startups que tienen entre seis y diez años de antigüedad se encuentran en la "etapa de crecimiento", en la que el factor material tiene mayor influencia en el crecimiento y desarrollo de la misma. b) Sector productivo

Al hacer un análisis por "sector productivo", como se observa en la Tabla 9, el 50\% (9) de las startups del sector "agricultura, pesca y apícola” y el 62.5\% (5) de las startups del sector "educación" se encuentran en el CS, es decir, en el que menor valor da a ambos componentes. Por el contrario, más del 50\% (17) de las startups del sector "industria y manufactura" y el $50 \%$ (4) de las startups de los sectores "comercio" y "suministro de agua, electricidad, vapor y gestión de residuos" se encuentran en el CI.

Tabla 9. Tabla cruzada "Sector Productivo" y Clústeres

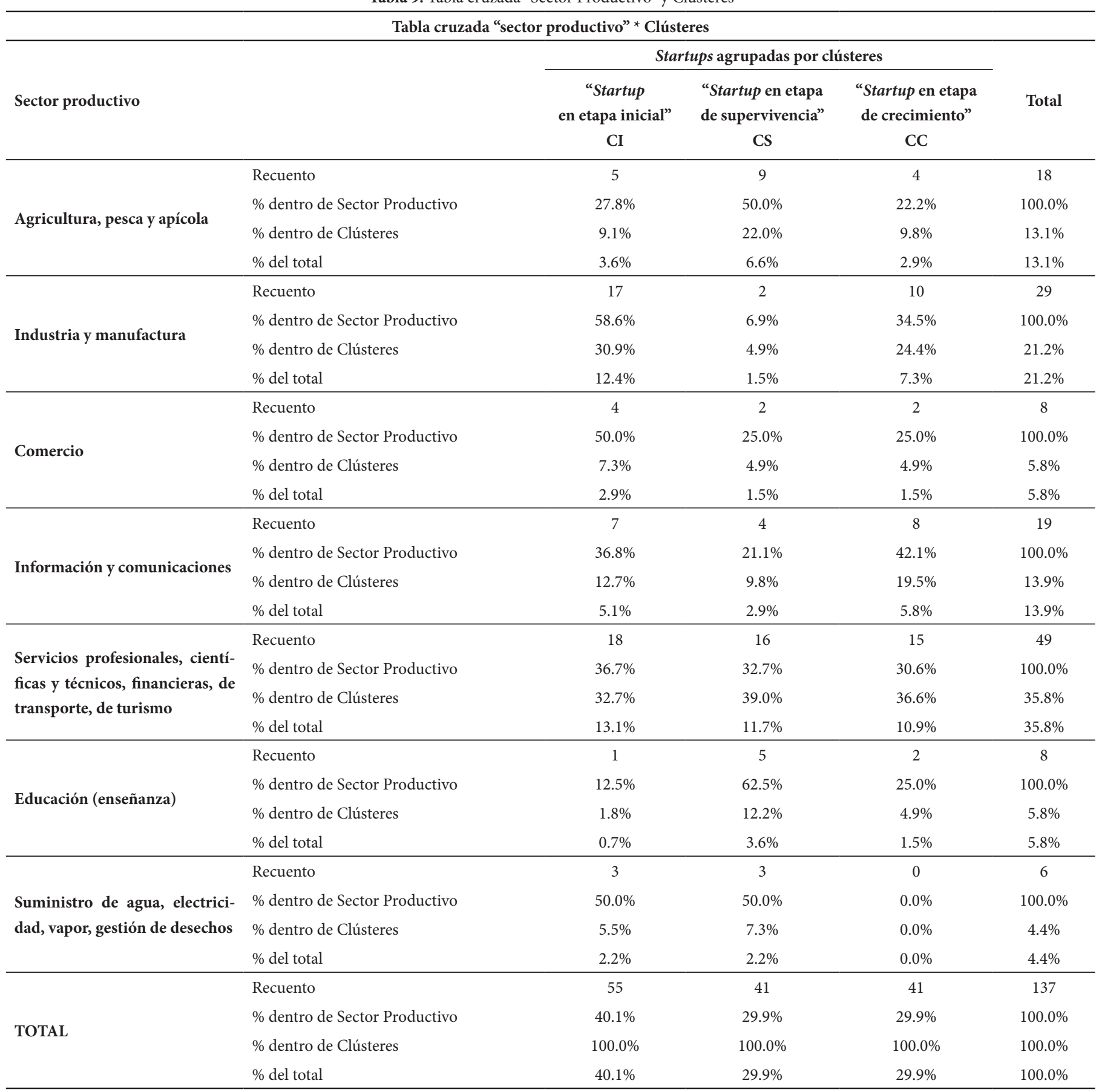


En el caso de las empresas del sector información y comunicaciones, el 42.1\% (8) pertenecen al CC en el que el factor material es más valorado, mientras que el $36.7 \%$ (18) de las empresas del sector servicios pertenecen al CI en el que ambos componentes son valorados. Cabe señalar que, en el sector servicios, las 49 startups se distribuyen casi uniformemente entre los tres clústeres.

\section{Discusión}

Los resultados muestran que, las acciones más valoradas por los emprendedores encuestados son: la existencia de un mercado atractivo, el acceso a tecnología y la existencia de fondos concursables. Esto coincide con otros estudios de la región (Kantis et al., 2018), según los cuales, en economías emergentes y ecosistemas incipientes, el acceso a una tecnología que permita desarrollar productos o servicios competitivos, el acceso a un mercado que permita crecer rápidamente de manera orgánica y el financiamiento del Estado en la etapa inicial del emprendimiento dinámico, son condiciones fundamentales para el crecimiento y desarrollo de startups, pues los fondos provenientes de la inversión ángel o capitales de riesgo, probablemente estén en fase de formación. Sin embargo, en ecosistemas de emprendimiento y economías con mayor nivel de desarrollo, el acceso a servicios de mentoría, financiamiento y networking son más valorados por los emprendedores (Spigel, 2015).

Por otra parte, la existencia de aceleradoras, incubadoras, inversionistas de alto riesgo y espacios de coworking, fueron poco valorados. No se conocen las razones de esta baja valoración, sin embargo, por ser un ecosistema de emprendimiento en etapa incipiente, es posible suponer que el conocimiento de los emprendedores, sobre estos actores y sus roles en el proceso de emprender, así como los vínculos entre ellos, sean limitados (Landström, Mason, \& Romaní, 2016; Mason \& Brown, 2014).

No obstante, como sostienen Brown y Mason (2017), en ecosistemas de emprendimiento embrionarios -como es el caso de Lima-, las acciones suelen estar orientadas a promover el emprendimiento y el surgimiento de nuevas iniciativas empresariales, más que a impulsar el crecimiento de las startups y la calidad de los emprendimientos. Por eso, se requiere un gran esfuerzo por parte del emprendedor, quien deberá validar su propuesta y conseguir los recursos necesarios para seguir creciendo a través del mercado, más que con el apoyo de redes de inversionistas ángeles, fondos públicos o alianzas con corporaciones. En consecuencia, es necesario que los gestores de programas e instrumentos para el desarrollo de ecosistemas de emprendimiento, tengan clara la distribución de startups por antigüedad, por sector al que pertenecen y la fase de emprendimiento en la que se encuentran, para que identifiquen los componentes que podrían incidir en su crecimiento (Spigel, 2015).

Con respecto a los componentes identificados, si se comparan los resultados con los atributos sociales y materiales planteados por Spigel (2015), de las ocho variables agrupadas en el componente I, cuatro (V5, V6, V7 y V8) corresponden al atributo "social" y cuatro (V1, V3, V4, y V11) al atributo "material"; mientras que en el componente II, las cuatro variables corresponden al atributo "material" planteado por Spigel (ver tabla 10). En ecosistemas incipientes, con recursos escasos, es de esperar que el acceso a algunos recursos, dependa más de la red de contactos del emprendedor que de la disponibilidad de recursos en el ecosistema.

Tabla 10. Comparación de los resultados del análisis de componentes principales con modelo de Spigel

\begin{tabular}{lll}
\hline Atributos Modelo de Spigel (2015) & Resultados ACP & Descripción de variable en estudio \\
\hline (M2) Servicios de apoyo e infraestructura & Social & (V3) La existencia de aceleradoras \\
(M2) Servicios de apoyo e infraestructura & Social & (V4) La existencia de incubadoras \\
(S2) Capital emprendedor & Social & (V5) La existencia de inversionistas de alto riesgo \\
(M2) Servicios de apoyo e infraestructura & Social & (V11) La existencia de coworkings gratuitos \\
(S3) Mentores y modelos a seguir & Social & (V8) Apoyo por parte de mentores \\
(S1) Redes sociales & Social & (V6) Visitas a otros ecosistemas internacionales \\
(S3) Mentores y modelos a seguir & Social & (V7) Apoyo por parte de otros emprendedores \\
(M3) Políticas y gobierno & Social & (V1) La existencia de fondos concursables \\
(M2) Servicios de apoyo e infraestructura & Material & (V12) Acceso a tecnología \\
(M1) Universidades & Material & (V10) Acceso a universidades y especialistas \\
(M3) Políticas y gobierno & Material & (V9) Acceso a laboratorios y centros de investigación \\
(M4) Mercados abiertos & Material & (V2) La existencia de un mercado atractivo
\end{tabular}
Social $=$ Componente $1 \quad$ Material $=$ Componente II

Con el fin de explorar si el financiamiento que el Estado estaba brindando a las incubadoras, aceleradoras, redes de inversionistas ángeles y fondos concursables, contribuía con el crecimiento y desarrollo de las startups, el atributo "servicios de apoyo e infraestructura" (M2) propuesto por Spigel se desagregó en el cuestionario. Los resultados mostraron muy bajas valoraciones de estas variables, lo que valdría la pena profundizar en estudios posteriores.

En un ecosistema de emprendimiento incipiente (Kantis et al., 2017), con reducido número de actores, pocos emprendimientos dinámicos 
e innovadores y falta de políticas de apoyo al emprendimiento dinámico, es de esperarse una baja valoración de los componentes. Sin embargo, los clústeres han evidenciado las distintas necesidades de los emprendedores en función a la fase de crecimiento y desarrollo en la que se encuentran. Cabe resaltar la importancia del componente material acceso a tecnología, universidades y especialistas, laboratorios y centros de investigación, además de la existencia de un mercado atractivo- para las startups en las etapas inicial y de crecimiento. En estas etapas, es probable que el acceso a recursos intangibles como el "conocimiento" -vinculado a la tecnología, talento, registros de patentes y oportunidades de mercado- tenga mayor influencia en el crecimiento y desarrollo de la startups, porque contribuyen directamente a su competitividad.

El análisis de clústeres y las tablas cruzadas permitió identificar que, en etapas iniciales, ambos componentes son relevantes para el crecimiento y desarrollo de startups. En este clúster la presencia de actores del ecosistema (incubadoras, aceleradoras, inversionistas, otros emprendedores, gobierno, academia) y el acceso a sus recursos (científicos, tecnológicos, financieros, conocimiento, talento y mercado) son valorados, especialmente por aquellos que están en los sectores de "industria y manufactura", "comercio", "servicios profesionales, científicos y técnicos, financieros, de transporte y de turismo" y "suministro de agua, electricidad, vapor y gestión de desechos".

Por el contrario, las 41 startups en etapa de supervivencia, valoran poco los atributos sociales y materiales, y los sectores con mayor representatividad en este clúster son "agricultura, pesca y apicultura" y "educación".

Con respecto a las 41 startups en "etapa de crecimiento" estas valoran el tributo material, mas no el social. Es probable que esto se deba a que $58.5 \%$ de startups tienen seis o más años de antigüedad, en cuyo caso, la existencia de incubadoras, aceleradoras, coworkings, inversionistas, fondos del Estado, visitas a ecosistemas de emprendimiento internacionales y el apoyo de mentores sea poco o nada valorado. Por el contrario, estas startups valoran el acceso a tecnología, universidades y especialistas, laboratorios y centros de investigación, así como la existencia de una demanda atractiva. Es posible suponer que, para estas startups, los recursos intangibles como el conocimiento y acceso a nuevos mercados, sea más valorado que el atributo social. Cabe resaltar que el único sector cuya mayor proporción de startups está en este clúster es el de "información y comunicaciones".

Con respecto a la caracterización de las startups en función al sector productivo, "educación” y "agricultura, pesca y apícola” son los que menor puntuación dieron a la influencia de los componentes -social y material-, mientras que las startups de los sectores "servicios profesionales, científicos, técnicos, financieros, de transporte y de turismo", "industria y manufactura" e "información y comunicaciones" son los que mayor valor dieron a ambos componentes. Estos resultados sugieren que el ecosistema de emprendimiento de Lima, por sus características económicas, geográficas, demográficas y socioculturales, probablemente aporte más al crecimiento y desarrollo de startups de los sectores secundario y terciario de la economía, que a otro tipo de sectores productivos.
Como sugieren Spigel y Harrison (2018), desde la perspectiva del desarrollo de los ecosistemas de emprendimiento, en una etapa inicial, los ecosistemas deberían brindar acceso a recursos materiales y apoyar en la vinculación entre los diversos actores, lo que es fundamental para el surgimiento y supervivencia de las startups. Sin embargo, en una etapa de mayor madurez del ecosistema, los diversos actores deberían ofrecer recursos para un crecimiento acelerado y un mayor desarrollo -productivo y competitivo- de las startups.

Finalmente, es importante evaluar si para países emergentes como Perú, con ecosistemas de emprendimiento incipientes, los programas o instrumentos de apoyo al desarrollo de ecosistemas de emprendimiento están favoreciendo el crecimiento acelerado y la mayor competitividad de startups, o más bien, están alargando la agonía de startups poco productivas y competitivas. En este sentido, las políticas de apoyo al emprendimiento y los programas de apoyo para la competitividad deberán considerar los recursos y capacidades de los emprendedores, de sus startups, de los diversos actores del ecosistema y el contexto más general -a nivel macro- que condiciona la competitividad de las startups.

\section{Conclusiones}

Para los formuladores y gestores de políticas públicas, es importante conocer y monitorear las necesidades de los emprendedores de startups, con el fin de que los escasos recursos públicos, sean asignados eficientemente y no se desperdicien tratando de reproducir condiciones o conceptos que han sido exitosos en otros contextos, bajo condiciones económicas, culturales, geográficas y sociodemográficas distintas.

Para el caso específico del ecosistema de emprendimiento de Lima, se requiere desarrollar el componente social, para llevar a las startups de una situación inicial de supervivencia, a un crecimiento acelerado. Pero también es necesario desarrollar el componente material, para acelerar el crecimiento y mejorar la productividad y competitividad de las startups, es decir, la calidad de los emprendimientos, más que la cantidad de los mismos.

En ecosistemas incipientes, de países emergentes, los programas de apoyo del Estado y un mercado atractivo contribuyen al crecimiento y desarrollo de startups, lo que coincide con el estudio de otros ecosistemas de emprendimiento en etapa embrionaria como Estonia (Saarenketo, Torkkeli, \& Velt, 2018). En este tipo de ecosistemas, si bien algunos actores podrían tener mayor incidencia que otros, como por ejemplo las instituciones del gobierno sobre las incubadoras, o un mercado atractivo sobre la existencia de redes de inversionistas ángeles, no hay un solo tipo de actor que dirija las actividades del ecosistema. Quizás por ello, la valoración de los emprendedores da como resultado una distribución no paramétrica de las variables estudiadas, lo que sugiere el nivel de desarrollo del ecosistema (Albort-Morant \& Oghazi, 2016). Sin embargo, la poca valoración dada a algunos actores, podría estar vinculada a la poca presencia o la baja calidad de sus servicios. 
En países emergentes como el Perú, con graves deficiencias en el sistema educativo, limitada plataforma de CTI e I+D y una cultura de innovación poco desarrollada (Kantis et al., 2018; OCDE, 2016), la competitividad de las startups es un reto, a pesar de contar con una fuerte cultura orientada al emprendimiento (Serida, Alzamora, Guerrero, Borda, \& Morales, 2018) y condiciones macroeconómicas y de mercado, favorables (Banco Central de Reserva del Perú, 2019). En estas circunstancias, se requiere un mayor desarrollo del componente material -acceso a la tecnología, acceso a universidades y especialistas, acceso a laboratorios y centros de investigación, y la existencia de un mercado atractivo-, pues el componente social -apoyo por parte de los diversos actores del ecosistema y acceso a financiamiento- por sí mismo no es suficiente. La incidencia del componente material en el crecimiento y desarrollo de startups evidencia la necesidad de diseñar incentivos y políticas que promuevan y estimulen la inversión -pública y privada- en actividades de innovación.

Finalmente, si bien se trata de un estudio exploratorio, los resultados brindan información relevante a los formuladores de políticas públicas y los gestores de programas e instrumentos de apoyo para startups. Se espera que esta información contribuya a la eficiencia del gasto público, y que los gobiernos regionales y locales "construyan" y "nutran" sus propios ecosistemas de emprendimiento, de acuerdo con la naturaleza específica de cada territorio y el perfil de sus emprendimientos (Feld, 2012), más que con "recetas exitosas" de otros contextos.

\section{Recomendaciones}

Se recomienda profundizar el estudio exploratorio incluyendo: i) un análisis de variables culturales, como la perseverancia y la ambición de los emprendedores o la disponibilidad de capital humano motivado a trabajar en una startup; y ii) ampliar la muestra a una mayor diversidad de emprendedores, no solo startups que tuvieron algún acercamiento al programa de financiamiento para el emprendimiento y la innovación de Innóvate Perú, sino también empresas de rápido crecimiento (High Growth Firms).

Se recomienda replicar el estudio en el resto de ciudades en las que Innóvate Perú ha otorgado fondos para el desarrollo de los ecosistemas de emprendimiento e innovación, como hizo previamente en la ciudad de Lima. Ello permitirá caracterizar las startups de cada localidad, comparar los resultados, hacer propuestas específicas y evaluar la eficiencia de los programas e instrumentos para el desarrollo de ecosistemas de emprendimiento en cada localidad. Sin embargo, se debe considerar que, en algunas zonas rurales, se podrían encontrar ciertas limitaciones de la comunidad para involucrarse en la construcción del ecosistema de emprendimiento, por falta de capacidades físicas, humanas y financieras. Ello podría limitar su construcción y desarrollo, y por lo tanto el apoyo del ecosistema a la competitividad de las startups se vería limitado.

\section{Implicancias para hacedores de políticas y administradores}

Conocer los componentes o atributos del ecosistema de emprendimiento de Lima que inciden en los tres clústeres identificados es importante y relevante para poder alinear el apoyo a las startups con una estrategia de transformación productiva y de innovación más amplia.

La incidencia del atributo material en el crecimiento y desarrollo de startups, evidencia la necesidad de diseñar incentivos y políticas que promuevan y estimulen la inversión -pública y privada- en actividades de innovación y en políticas de innovación y desarrollo productivo. En este sentido, es necesario alinear las políticas de: fomento para startups, fortalecimiento de los actores y desarrollo de ecosistemas de emprendimiento, con políticas de innovación y transformación productiva, pues las probabilidades de éxito de una startup dependen de las capacidades para innovar y emprender, así como de las relaciones entre todos los actores de un distrito industrial o ecosistema.

Reconocer la importancia de las startups para el progreso económico y bienestar social de una localidad, implica reconocer que para medir su aporte se deben usar métricas distintas al número de empresas que surgen o mueren. Indicadores como tasa mensual de crecimiento en ingresos o número de clientes, incursión en nuevos mercados, desarrollo de nuevos productos, número de registros de propiedad intelectual y nivel de satisfacción de los clientes o usuarios, suelen ser más eficientes para medir el nivel de innovación y escalabilidad de las startups. Además, es recomendable establecer sistemas de acreditación de la calidad de los servicios ofrecidos por los diversos actores, en función al impacto en la startup. De esta manera, se estaría evitando que los actores del ecosistema se conviertan en "captadores de recursos" con baja eficiencia.

El ecosistema de apoyo a las startups de Lima está pasando de una etapa de "gestación y experimentación" con una lógica de "piloto", a una etapa de "consolidación o expansión" con una lógica de "escalamiento" en Lima y otras regiones (OCDE, 2016). Por ello, requiere de programas de apoyo ágiles y dinámicos, capaces de dialogar con inversionistas y empresarios; mientras que el sistema de innovación, que dialoga con investigadores, académicos y corporaciones, suele ser menos ágil (OCDE 2017). En general, en Latinoamérica, el dinamismo de las políticas, programas e instrumentos de apoyo para de desarrollo de ecosistemas de emprendimiento ha sido mayor que las intervenciones más tradicionales de apoyo a la innovación y competitividad (OECD, 2016a).

Las probabilidades de éxito de una startup dependen de las capacidades para innovar y emprender, y de las relaciones entre los actores de un clúster o ecosistema, que la ayuden a elegir e implementar una estrategia (Murray \& Stern, 2015). Por ello, fortalecer la cultura empresarial y la cultura de innovación son aspectos fundamentales para el crecimiento y desarrollo de las startups. 


\section{Acerca del autor:}

Karen Edith Weinberger Villarán, profesora principal e investigadora de la Universidad del Pacífico. Especialista en emprendimiento y responsable de la cátedra de emprendimiento del departamento académico de administración, de la Facultad de Ciencias Empresariales. Fundó el Centro de Emprendimiento e Innovación, Emprende UP. Entre 2016 y 2018, participó en el MIT Regional Entrepreneurship Acceleration Program - Lima, cohort 4, en representación de la academia. Actualmente es miembro de la red de inversionistas ángeles The Board, miembro alterno de la Mesa Ejecutiva de Innovación del Ministerio de Economía y Finanzas y miembro del Consejo de Directores de Global Entrepreneurship Network - Perú.

\section{Agradecimientos:}

A Innóvate Perú por las facilidades otorgadas para el levantamiento de información; al equipo del MIT REAP Lima - cohort 4 por los dos años de trabajo en favor del desarrollo del ecosistema de emprendimiento de Lima; a los profesores Luis Camilo Ortigueira, Angie Higuchi, Enrique Saravia, y a mi asistente Gabriela Castillo, por sus aportes en la presente investigación.

\section{Referencias}

Ács, Z., Autio, E., \& Szerb, L. (2014). National Systems of Entrepreneurship: Measurement issues and policy implications. Research Policy, 43(3), 476-494. https://doi.org/https://doi.org/10.1016/j.respol.2013.08.016

Albort-Morant, G., \& Oghazi, P. (2016). How useful are incubators for new entrepreneurs? Journal of Business Research, 69(6), 21252129. https://doi.org/10.1016/j.jbusres.2015.12.019

Alvedalen, J., \& Boschma, R. (2017). A critical review of entrepreneurial ecosystems research: towards a future research agenda. European Planning Studies, 25(6), 887-903. https://doi.org/10.1080/09654313. 2017.1299694

Auerswald, P. E., Auerswald, P. P., Acs, Z., Florida, R., Schramm, C., Stangler, D., ... Stefanotti, J. (2015). Enabling Startup Ecosystems. (October).

Aulet, B. (2008). How to build a successful innovation ecosystem: education, network and celebrate.

Banco Central de Reserva del Perú. (2019). Memoria 2018. Retrieved from http://www.bcrp.gob.pe/docs/Publicaciones/Memoria/2018/ memoria-bcrp-2018.pdf

Blank, S. (2003). The four steps to the epiphany: Successful strategies for products that win. Pescadero: K\&S Ranch.

Bosma, N., \& Kelley, D. (2019). Global Entrepreneurship Monitor 2018/2019 Global Report.
Brown, R., \& Mason, C. (2017). Looking inside the spiky bits: a critical review and conceptualisation of entrepreneurial ecosystems. Small Business Economics, 49(1), 11-30. https://doi.org/10.1007/ s11187-017-9865-7

Brown, R., Mawson, S., \& Mason, C. (2017). Myth-busting and entrepreneurship policy: the case of high growth firms. Entrepreneurship \& Regional Development, 29(5-6), 414-443. https://doi.org/10.1080/089 85626.2017 .1291762

Clarysse, B., Wright, M., Bruneel, J., \& Mahajan, A. (2014). Creating value in ecosystems: Crossing the chasm between knowledge and business ecosystems. Research Policy, 43(7). https://doi.org/10.1016/j. respol.2014.04.014

Cohen, B. (2006). Sustainable valley entrepreneurial ecosystems. Business Strategy and the Environment, 15(1), 1-14.

Feld, B. (2012). Startup communities: building an entrepreneurial ecosystem in your city. John Wiley \& Sons.

Fetters, M., Greene, P. G., \& Rice, M. P. (2010). The development of university-based entrepreneurship ecosystems: Global practices. Edward Elgar Publishing.

Finger, M., \& Samwer, O. (1998). America's most successful startups: Lessons for entrepreneurs. Springer Science \& Business Media.

Fornell, C. (1981). A comparative analysis of two structural equation models: LISREL and PLS applied to market data.

Gnyawali, D. R., \& Fogel, D. S. (1994). Environments for entrepreneurship development: key dimensions and research implications. Entrepreneurship Theory and Practice, 18(4), 43-62.

Hair Jr, J. F., Anderson, R. E., Tatham, R. L., \& Black, W. C. (1999). Análisis multivariante. Madrid: Prentice Hall.

Hernández, C., \& González, D. (2016). Study of the Start-Up Ecosystem in Lima, Peru: Collective Case Study. Latin American Business Review, 17(2), 115-137. https://doi.org/https://doi.org/10.1080/1097 8526.2016 .1171678

Instituto Nacional de Estadística e Informática. (2018). Perú: Perfil Sociodemográfico Informe Nacional.

Instituto Nacional de Estadística e Informática. (2019a). Evolución de la Pobreza Monetaria 2007-2018. Lima.

Instituto Nacional de Estadística e Informática. (2019b). Perú: Evolución de los Indicadores de Empleo e Ingreso por Departamento, 2007-2018. Lima.

Isenberg, D. J. (2010). How to Start an Entrepreneurial Revolution. Harvard Business Review, 88(6), 40-50. Retrieved from https://institute.coop/sites/default/files/resources/Isenberg - How to Start an Entrepreneurial Revolution.pdf 
Isenberg, D. J. (2011). Introducing the Entrepreneurship Ecosystem: Four Defining Characteristics. Forbes. Retrieved from https://www. forbes.com/sites/danisenberg/2011/05/25/introducing-the-entrepreneurship-ecosystem-four-defining-characteristics/\#733a7ebb5fe8

Isenberg, D., \& Onyemah, V. (2016). Fostering scaleup ecosystems for regional economic growth (innovations case narrative: ManizalesMas and Scale Up Milwaukee). Innovations: Technology, Governance, Globalization, 11(1-2), 60-79. Retrieved from https://www.mitpressjournals.org/doi/pdf/10.1162/inov_a_00248

Kantis, H., Federico, J., \& Ibarra García, S. (2015). Condiciones Sistémicas para el Emprendimiento Dinámico: América Latina en el nuevo escenario global. Retrieved from https://www.fomin.org/Portals/0/ Publicaciones/ICSEd_2015.pdf

Kantis, H., Federico, J., \& Ibarra García, S. (2017). Condiciones Sistémicas para el Emprendimiento Dinámico 2017. América Latina: avances y retrocesos en perspectiva. Retrieved from https://prodem.ungs. edu.ar/publicaciones_prodem/condiciones-sistemicas-para-el-emprendimiento-dinamico-2017-america-latina-avances-y-retrocesosen-perspectiva/

Kantis, H., Federico, J., \& Ibarra García, S. (2018). Condiciones sistémicas para el emprendimiento dinámico. Las brechas abiertas de América Latina: ¿convergencia o divergencia?

Kantis, H., Federico, J., Ibarra García, S., \& Menéndez, C. (2015). Estudio de las condiciones para el emprendimiento dinámico e innovador en Perú y sus principales barreras. Retrieved from https:// docplayer.es/17255119-Estudio-de-las-condiciones-para-el-emprendimiento-dinamico-e-innovador-en-peru-y-sus-principales-barreras.html

Kelley, D. J., Singer, S., \& Herrington, M. (2012). Global Entrepreneurship Monitor: Global Report 2011. Babson College, Universidad Del Desarrollo, Universiti Tun Abdul Razak, 38. Retrieved from http:// scholar.google.com/scholar?hl=en\&btnG=Search\&q=intitle:Global+ Entrepreneurship+Monitor+2011+Global+Report\#8

Landström, H., Mason, C., \& Romaní, G. (2016). Business angels in developing economies: the experience of Latin America. Handbook of Research on Business Angels, (October), 282-324. https://doi. org/10.4337/9781783471720.00019

Lopez, T., \& Alvarez, C. (2018). Entrepreneurship research in Latin America: a literature review. Academia Revista Latinoamericana de Administracion. https://doi.org/10.1108/ARLA-12-2016-0332

Mack, E., \& Mayer, H. (2016). The evolutionary dynamics of entrepreneurial ecosystems. Urban Studies, 53(10). https://doi. org/10.1177/0042098015586547

Malecki, E. J. (2018). Entrepreneurship and entrepreneurial ecosystems. Geography Compass, 12(3). https://doi.org/https://doi. org/10.1111/gec3.12359
Mason, C., \& Brown, R. (2014). Entrepreneurial Ecosystems and Growth Oriented Entrepreneurship. Retrieved from https://www.oecd. org/cfe/leed/Entrepreneurial-ecosystems.pdf

Mike Herrington and Penny Kew, G. E. M. (2016). Global Entrepreneurship Monitor 2016/2017. Global Entrepreneurship Monitor. https://doi.org/10.1017/CBO9781107415324.004

Ministerio de la Producción. (2014). Plan Nacional de Diversificación Productiva.

Ministerio de la Producción. (2015). Innóvate Perú: caja de herramientas para la innovación (Primera; Ministerio de la Producción, Ed.). Lima - Perú.

Ministerio de la Producción. (2019). Diagnóstico Innóvate Perú.

Motoyama, Y., \& Knowlton, K. (2016). Examining the Connections within the Startup Ecosystem: A Case Study of St. Louis. Entrepreneurship Research Journal, 7(1). https://doi.org/10.1515/erj-2016-0011

Murray, F., \& Stern, S. (2015). Linking and Leveraging. Science, 348(6240), 1203. https://doi.org/https://doi.org/10.1126/science. aac5843

Neck, H. M., Mayer, G. D., Cohen, B., \& Corbett, A. C. (2004). An entrepreneurial system view of new venture creation. Journal of Small Business Management, 42(2), 190-208.

Nicotra, M., Romano, M., Del Giudice, M., \& Schillaci, C. E. (2018). The causal relation between entrepreneurial ecosystem and productive entrepreneurship: a measurement framework. Journal of Technology Transfer, 43(3), 640-673. https://doi.org/10.1007/s10961-017-9628-2

Nunnally, J. (1994). Psychometric methods (3rd ed.). Tata McGrawHill Education.

OCDE. (2016). Startup América Latina: Promoviendo la innovación en la región (OECD Publishing, Ed.).

OECD. (2016a). Start-up Latin America 2016 Building an Innovative Future: Why support start-ups, and how? https://doi.org/https://doi. org/10.1787/9789264265660-6-en

OECD. (2016b). Start up Latin America 2016: Building an Innovative Future.

Olutuase, S. O., Brijlal, P., Yan, B., \& Ologundudu, E. (2018). Entrepreneurial Orientation and Intention: Impact of Entrepreneurial Ecosystem Factors. Journal of Entrepreneurship Education, 21(3), 1-14. Retrieved from https://www.abacademies.org/articles/Entrepreneurial-orientation-and-intention-1528-2651-21-3-185.pdf

Qian, H. (2018). Knowledge-Based Regional Economic Development: A Synthetic Review of Knowledge Spillovers, Entrepreneurship, and Entrepreneurial Ecosystems. Economic Development Quarterly, 32(2), 163-176. https://doi.org/10.1177/0891242418760981 
Roberts, P. W., Thomas, E., Chao, C., Edens, G., Davidson, A., Heidkamp, K., \& Yeo, J.-H. (2017). Accelerating Startups in Emerging Markets: Insights from 43 Programs.

Roundy, P. T., Bradshaw, M., \& Brockman, B. K. (2018). The emergence of entrepreneurial ecosystems: A complex adaptive systems approach. Journal of Business Research, 86, 1-10. https://doi. org/10.1016/j.jbusres.2018.01.032

Roundy, P. T., Brockman, B. K., \& Bradshaw, M. (2017). The resilience of entrepreneurial ecosystems. Journal of Business Venturing Insights, 8(11), 99-104. https://doi.org/10.1016/j.jbvi.2017.08.002

Saarenketo, S., Torkkeli, L., \& Velt, H. (2018). Uncovering new value frontiers: the role of the entrepreneurial ecosystem in nurturing born globals. International Journal of Export Marketing. https://doi. org/10.1504/ijexportm.2018.10020582

Serida, J., Alzamora, J., Guerrero, C., Borda, A., \& Morales, O. (2016). Global Entrepreneurship Monitor Perú 2015-2016. Retrieved from https://www.esan.edu.pe/publicaciones/2016/12/15/reporte_GEM 2015-2016 final.pdf

Serida, J., Alzamora, J., Guerrero, C., Borda, A., \& Morales, O. (2018). Global Entrepreneurship Monitor 2017-2018.

Serida, J., Guerrero, C., Alzamora, J., Borda, A., \& Morales, O. (2017). Global Entrepreneurship Monitor Perú 2016-2017. Retrieved from https://www.esan.edu.pe/publicaciones/2017/10/12/GEM 2016-2017 VF.pdf

Shane, S., \& Venkataraman, S. (2000). The Promise of Entrepreneruship as a Field of Research. The Academy of Management Review, 25(1), 217-226. https://doi.org/10.2307/259271

Skala, A. (2019). The Startup as a Result of Innovative Entrepreneurship. In Digital Startups in Transition Economies (pp. 1-40). Palgrave Pivot, Cham.

Spender, J. C., Corvello, V., Grimaldi, M., \& Rippa, P. (2017). Startups and open innovation: a review of the literature. European Journal of Innovation Management. https://doi.org/10.1108/EJIM-12-20150131
Spigel, B. (2015). The Relational Organization of Entrepreneurial Ecosystems. Entrepreneurship Theory and Practice, 41(1), 49-72. https://doi.org/10.1111/etap.12167

Spigel, B., \& Harrison, R. (2018). Toward a process theory of entrepreneurial ecosystems. Strategic Entrepreneurship Journal, 12(1), 151168. https://doi.org/10.1002/sej.1268

Spilling, O. R. (1996). The entrepreneurial system: On entrepreneurship in the context of a mega-event. Journal of Business Research, 36(1), 91-103. https://doi.org/10.1016/0148-2963(95)00166-2

Stam, E., \& Spigel, B. (2016). Entrepreneurial ecosystems. USE Discussion Paper Series, 16(13).

Theodoraki, C., \& Messeghem, K. (2017). Exploring the entrepreneurial ecosystem in the field of entrepreneurial support: a multi-level approach. International Journal of Entrepreneurship and Small Business, 31(1). https://doi.org/10.1504/ijesb.2017.083847

Tripathi, N., Oivo, M., Liukkunen, K., \& Markkula, J. (2019). Startup ecosystem effect on minimum viable product development in software startups. Information and Software Technology. https://doi. org/10.1016/j.infsof.2019.06.008

Tripathi, N., Seppänen, P., Boominathan, G., Oivo, M., \& Liukkunen, K. (2019). Insights into startup ecosystems through exploration of multi-vocal literature. Information and Software Technology, 105, 56-77. https://doi.org/10.1016/j.infsof.2018.08.005

Vogel, P. (2013). The Employment Outlook for Youth: Building Entrepreneurial Ecosystems as a Way Forward. G20 Youth Forum, 443-449. Retrieved from http://ssrn.com/abstract $=2357856$

Welter, F., Baker, T., Audretsch, D. B., \& Gartner, W. B. (2017). Everyday Entrepreneurship-A Call for Entrepreneurship Research to Embrace Entrepreneurial Diversity. Entrepreneurship Theory and Practice, 41(3), 311-321. https://doi.org/10.1111/etap.12258

Yin, R. K. (2013). Applications of case study research. Applied Social Research Methods Series, 34, 173. https://doi.org/10.1097/ FCH.0b013e31822dda9e 
